\title{
ARTIGOS
}

\section{EL ESTATUS EPISTÉMICO DE LOS EXPERIMENTOS MENTALES EN CIENCIAS FACTICAS*}

\author{
Bruno Borge** \\ brunojborge@gmail.com \\ Guadalupe Mettini*** \\ guadalupemettini@gmail.com
}

RESUMEN Un experimento mental en ciencias fácticas consiste en la representación de un escenario imaginario. A partir de la presentación de condiciones iniciales y la postulación de una situación hipotética o contrafáctica, se solicita al lector que realice mentalmente alguna operación, manipule ciertas variables o ponga en funcionamiento algún aparato o instrumento. En virtud de este ejercicio sería posible, en principio, obtener nuevo conocimiento acerca de algún aspecto del mundo natural. El debate acerca de las funciones de los experimentos mentales en las ciencias fácticas está centrado en establecer la validez del conocimiento acerca del mundo empírico que estos dispositivos son capaces de proporcionar. La propuesta interpretativa de Brown (1991, 2011) da sustento a la idea de que, en casos excepcionales, los experimentos mentales pueden cumplir funciones probatorias. Los denominados "experimentos mentales platónicos" permiten intuir leyes de la naturaleza y justificar a priori las hipótesis propuestas. Sostenemos que la conexión trazada por Brown entre los experimentos mentales y leyes naturales exhibe un aspecto problemático de su filosofia: el compromiso injustificado con una metafísica inflacionaria.

* Artigo submetido em 12/06/17. Aceito em 01/08/17.

** Universidad de Buenos Aires / IIF-SADAF-CONICET. Buenos Aires - Argentina.

*** Universidad de Buenos Aires. Buenos Aires - Argentina. 
Mostramos que es posible explicar el mecanismo a través del cual estos dispositivos proporcionan conocimiento nuevo y dar cuenta tanto de su poder heurístico cómo de su valor evidencial a partir compromisos metafísicos más modestos.

Palabras clave Experimentos mentales, leyes de la naturaleza, conocimiento a priori, inferencia a la mejor explicación, disposicionalismo.

ABSTRACT Thought experiments in factual science consists on the representation of an imaginary scenario. Starting with the presentation of a hypothetical or counterfactual situation and the description of initial conditions, the reader is asked to mentally perform some operation, to manipulate certain variables or to put into operation some apparatus or instrument. In virtue of this exercise, it would be possible to obtain new knowledge about the natural world. The debate on the functions of thought experiments in physics is focused on determining the validity of the empirical knowledge that these devices are capable of providing. Brown $(1991,2011)$ supports the idea that, in exceptional cases, thought experiments can perform probative functions. The so-called "Platonic Thought Experiments" allow us to intuit laws of nature and provide an a priori justification of the proposed hypotheses. We hold that Brown's connection between thought experiments and laws of nature exhibits a problematic aspect of his philosophy: the unjustified commitment with an inflationary metaphysics. We show that it is possible to explain the mechanism through which these devices provide new knowledge and give an account of both their heuristic power and their evidential value from modest metaphysical commitments.

Keywords Thought Experiments, Laws of Nature, A priori Knowledge, Inference to the Best Explanation, Dispositionalism.

\section{Introducción}

El debate acerca de las funciones de los experimentos mentales en las ciencias fácticas ${ }^{1}$ está centrado en establecer la validez del conocimiento acerca del mundo empírico que estos dispositivos son capaces de proporcionar. Debido a que esta clase de experimentos no introduce información empírica nueva, el 
examen epistémico de su funcionamiento debe incluir además una explicación acerca del origen de este conocimiento.

Uno de los aspectos más interesantes de esta discusión se vincula a las funciones probatorias que puedan desempeñar. En caso de que estos dispositivos posean valor evidencial o puedan cumplir algún papel relevante en la elección de teorías una explicación completa de sus poderes epistémicos debe dar cuenta de cómo es posible que proporcionen evidencia para una nueva teoría sin intervenir en el mundo natural.

La propuesta interpretativa de Brown da sustento a la idea de que en casos excepcionales los experimentos mentales posibilitan la revisión de teorías y aumentan el grado de creencia racional en una nueva hipótesis postulada. Hacen esto en virtud de la presentación de fenómenos, concebidos sólo en la imaginación, que ejemplifican clases naturales. La propiedad de exhibir este tipo de fenómenos es la que permite abreviar el salto inductivo que va desde las instancias particulares a la generalidad de la ley. Por consiguiente, sostiene Brown, esta clase particular de experimentos mentales nos permite intuir leyes de la naturaleza y justificar a priori las hipótesis propuestas.

La perspectiva de Brown da sentido a la intuición de acuerdo los experimentos mentales son esencialmente experimentos. Esta línea interpretativa da cuenta del valor evidencial de estos dispositivos y explica, al menos en parte, su importancia histórica en relación al papel que han desempeñado en la elección de teorías. Por otra parte, esclarece su valor heurístico, distinguiendo las operaciones de visualización de las inferencias lógicas y explicita el proceso a través del cual es posible obtener conocimiento nuevo. No obstante sostenemos que la conexión trazada por Brown entre los experimentos mentales y las leyes naturales exhibe un aspecto problemático de su filosofía: el compromiso injustificado con una metafísica inflacionaria, dado que es posible proporcionar una explicación de los poderes epistémicos de los experimentos mentales que haga justicia a su carácter experimental asumiendo compromisos epistémicos más modestos. Intentaremos mostrar que los procesos de idealización involucrados en la construcción de experimentos mentales permiten capturar la estructura disposicional de las entidades y procesos involucrados mediante una inferencia a la mejor explicación. Todo ello sin postular la existencia de leyes que gobiernen sus instancias.

\section{2 ¿Qué es realmente un experimento mental?}

El problema epistemológico de los experimentos mentales consiste en determinar las funciones que éstos pueden desempeñar respecto de hipótesis y teorías y en establecer las fuentes del conocimiento acerca del mundo natural 
que son capaces de proveer. El tratamiento sistemático de esta cuestión ha dado lugar a varias propuestas explicativas. Dos son las perspectivas antagónicas fundamentales: la experimental y la argumental. De acuerdo con la primera, representada fundamentalmente por Sorensen (1992) y Brown (1991, 2011), los experimentos mentales son capaces de cumplir las mismas funciones que los experimentos reales prescindiendo de la ejecución material. A partir de la narrativa de una situación hipotética o contrafáctica, que incluye información acerca de las condiciones iniciales y, en ocasiones, la descripción del funcionamiento de algún aparato, sería posible arribar a un resultado experimental. La segunda, representada principalmente por Norton (1991, 1996, 2004a, 2004b) e Irvine (1996) asume que dado que la intervención en el mundo material es esencial en la experimentación y los experimentos mentales son construidos y ejecutados solo en la imaginación, sus poderes epistémicos se reducen a la reorganización de información procedente de la experiencia.

El análisis sistemático de los poderes epistémicos de los experimentos mentales en el contexto de justificación de las teorías físicas se desarrolló a partir de los trabajos de Brown (1991, 2004a, 2004b, 2011) y Sorensen (1992). Estas obras inaugurales asumen que existe una continuidad entre experimentos reales y mentales. No obstante, las perspectivas difieren en cuanto a las tesis metafísicas y epistémicas que suscriben. Para Sorensen (1992, p. 205), los experimentos mentales son una subclase de experimentos reales no ejecutados, resultado de un proceso de evolución de las prácticas experimentales. El apoyo evidencial que los experimentos mentales pueden brindar depende de cómo reorganizan información empírica ya disponible en el sistema de creencias del experimentador. En el contexto de justificación los experimentos mentales cumplen principalmente funciones negativas, ${ }^{2}$ compeliendo al experimentador al abandono de una teoría que resulta implausible en el contexto de un escenario contrafáctico. De acuerdo con Brown (2011, p. 1) las similitudes entre experimentos mentales y reales permiten establecer una continuidad entre ambos. Como en los experimentos reales, el experimentador desempeña un rol activo. El relato del experimento mental incluye una serie de indicaciones que el experimentador debe seguir para ver qué sucede. Asimismo la ejecución de los de los experimentos mentales está ligada una actividad de visualización, que cumple una función análoga a la que desempeña la observación en los experimentos reales. Para Brown los experimentos mentales pueden, como los experimentos reales, ilustrar teorías,

2 La principal diferencia entre las perspectivas de Brown y Sorensen se encuentra en este punto. Para Sorensen los experimentos mentales exitosos funcionan como refutadores aléticos y su estructura corresponde con la de una paradoja, aunque su ejecución no se reduce a la de un argumento (Sorensen, 1992, p. 135). 
simular fenómenos y refutar o confirmar conjeturas científicas y en algunos casos incluso probar teorías. Los que denomina "experimentos mentales platónicos" poseen poderes epistémicos extraordinarios:

Un experimento mental platónico es un experimento que destruye una teoría existente o antigua y simultáneamente genera una nueva; es a priori porque no está basado en nueva evidencia empírica y no es una inferencia lógica cuya conclusión se deriva de viejos datos; representa además un avance en tanto la teoría resultante es mejor que su predecesor (Brown, 2011, p. 99).

El ejemplo más notable de un experimento de este tipo es el realizado por Galileo en sus "Discorsi” (1638 [1954, pp. 108-116]) con el objetivo de exhibir contradicciones en la física aristotélica. Galileo se representa dos móviles cuyas velocidades naturales son diferentes. De acuerdo con Aristóteles los cuerpos de mayor peso caen a una velocidad mayor que los cuerpos de menor peso. ¿Qué sucedería, según la descripción de Aristóteles, si los dos cuerpos de diferente peso fueran unidos en un compuesto por una cuerda de peso despreciable y liberados desde cierta altura? ¿El compuesto de un cuerpo de mayor peso y uno de menor peso caería más rápido o más lento que el cuerpo de mayor peso liberado individualmente? Según el razonamiento de Aristóteles, si el cuerpo más liviano cae más lento, entonces, retardará la caída del más pesado, por lo que el sistema compuesto por ambos caerá más lento que el cuerpo más pesado solo. Por otro lado, el sistema que combina los dos cuerpos es más pesado que el cuerpo más pesado aisladamente y, por consiguiente, debería caer más rápido que éste. La conclusión a la que se arriba siguiendo el razonamiento aristotélico es que el sistema combinado es al mismo tiempo más lento y más rápido que el cuerpo más pesado considerado individualmente, lo cual es evidentemente contradictorio. Para Galileo, la manera de resolver la paradoja consiste en suponer que tanto el sistema combinado como el cuerpo más pesado individualmente caen a la misma velocidad. El experimento establece el principio, que después sería conocido como "principio de equivalencia", de acuerdo con el cual todos los cuerpos caen con la misma velocidad independientemente de su masa y composición. Para Brown la ejecución de un experimento como este puede simultáneamente mostrar la inconsistencia de una teoría y establece una nueva en virtud de nuestra facultad de intuir leyes de la naturaleza.

Norton, el principal representante de la perspectiva argumental, afirma que dado que la intervención activa en el mundo material es lo que define a las prácticas experimentales y solo en virtud de ésta podemos ampliar nuestro conocimiento al respecto, los experimentos mentales son formas de razonamiento científico diferentes de la experimentación. Las reconstrucciones o reinterpretaciones de los datos de la experiencia que ocurren en los experimentos mentales y 
que pudieran contribuir en revisiones de creencias se explican a partir de los mecanismos de inferencia lógica tradicionales.

A pesar de su exótica reputación los experimentos mentales nos convencen a través de métodos bastante prosaicos. Llegan a nosotros en palabras en un papel. [...] Nos convencen sin experiencias exóticas, momentos bíblicos o estados mentales extáticos. A este nivel de descripción los experimentos mentales no difieren de la literatura en general (Norton, 2004a, p. 51).

Según Norton, la única alternativa real $^{3}$ a esta explicación es postular que estos dispositivos son capaces de realizar una especie de "magia epistémica" (Norton, 2004a, p. 44) al proveernos de conocimiento acerca del mundo natural del mismo modo que los experimentos reales pero omitiendo su ejecución. La apariencia experimental de la narrativa de escenarios hipotéticos, puede ser eliminada sin ninguna pérdida en cuanto a su alcance epistémico dado que se limita a la validez de la inferencia y al valor de verdad de las premisas. La mayoría de los experimentos mentales con fines refutatorios, tienen la forma de una reducción al absurdo. En estos casos la narrativa describe un escenario que enuncia un contraejemplo para una afirmación universalmente cuantificada. De esto se sigue una contradicción que implica la negación de un enunciado legal (Norton, 1991, p. 132). Muchos de los experimentos mentales empleados para apoyar teorías, se caracterizan por incluir inferencias inductivas. Este tipo de experimentos suele incluir una premisa implícita que afirma que el caso aludido es un caso típico y que los particulares referidos son inesenciales por lo que la conclusión se sostiene también para otros casos (Norton, 1991, p. 131).

Norton sostiene que, si fuera posible reconstruir todos los experimentos mentales como argumentos, entonces su interpretación sería verificada. El famoso experimento de Galileo, tiene para Norton la estructura de una reducción al absurdo:

1. Supuesto I para la prueba por reducción al absurdo: la velocidad de caída de los cuerpos en un medio dado depende de su peso.

2. De este supuesto se sigue que un cuerpo de mayor peso cae a una velocidad mayor que un cuerpo de menor peso.

3 Recientemente Buzzoni (2010) ha explorado la tesis de la continuidad entre experimentos reales y mentales a partir una epistemología kantiana. Esta perspectiva no es discutida por Norton ni tampoco por Brown. Presenta algunas similitudes con la perspectiva de Sorensen en cuanto afirma la tesis de la continuidad, pero se centra en la naturaleza lingüística de estos dispositivos, en tanto examina el funcionamiento de los conceptos involucrados en su ejecución. La fertilidad de esta perspectiva para establecer una tercera posición depende fundamentalmente de renunciar a la tesis que afirma naturaleza experimental de estos dispositivos, hipótesis que intentamos defender en este artículo. 
3. Si un cuerpo más lento es conectado a un cuerpo más rápido, entonces, el más lento retardará al más rápido y el más rápido acelerará al más lento.

4. Supuesto II: el compuesto es más pesado que los dos cuerpos separadamente.

5. Se sigue de 3 que si dos cuerpos de distinto peso son conectados, entonces, el compuesto caerá más rápido que el cuerpo más pesado individualmente.

6. Se sigue de 3 que si dos cuerpos de distinto peso son conectados, entonces, el compuesto caerá más lento que el cuerpo más pesado individualmente

7. 5 y 6 se contradicen.

8. Rechazamos el supuesto I.

9. Conclusión: todos los cuerpos caen con la misma velocidad independientemente de su peso.

Según Brown reconocer que es posible reconstruir experimentos mentales como argumentos no representa un obstáculo para sostener que son esencialmente experimentos. En este caso, la reconstrucción de Norton le permite mostrar su punto: algunos experimentos mentales trascienden el empirismo, nos permiten captar a priori leyes de la naturaleza. El resultado del experimento, que Norton formula en la conclusión, no puede seguirse de la contradicción resultado de la reductio, ya que cualquier conclusión puede seguirse de ella. El principio de equivalencia es una ley natural que intuimos al ejecutar el experimento.

El planteo de las propuestas en controversia nos permite realizar una evaluación preliminar de sus poderes explicativos. El punto de vista argumental representa una perspectiva deflacionaria respecto del funcionamiento epistémico de los experimentos mentales. Como tal cuenta con algunas ventajas, fundamentalmente proporciona una explicación clara y apoyada en la reconstrucción de casos del funcionamiento de estos dispositivos, además de ser austera respecto de los compromisos metafísicos que asume. Por otra parte, acomoda el funcionamiento de estos dispositivos a la epistemología empirista, dominante en la filosofía de la ciencia. No obstante, es limitada respecto de varios aspectos importantes. En primer lugar no hace justicia a la intuición de acuerdo con la cual los experimentos mentales son eventos o procesos en lugar de premisas y mecanismos de inferencia. En segundo lugar el conocimiento nuevo que proporciona la realización de algunos experimentos mentales parece no limitarse a la reorganización conceptual que tiene lugar en el experimento mental. Por el contrario, la perspectiva experimental, representada por Brown da sentido a ciertas intuiciones acerca del funcionamiento de los experimentos mentales y da cuenta de su importancia histórica. Parte importante de la fuerza persuasiva de los experimentos mentales se debe a que en ellos la participación 
del experimentador es un rasgo esencial. Desde la perspectiva de Brown, la representación de un escenario imaginario y la manipulación dentro de éste ce ciertas variables, permite caracterizar a los mismos como experimentos. De estas operaciones depende su eficacia o valor heurístico. Asimismo su significación evidencial parece estar asociada al tipo de fenómenos que en ellos se presentan más que a la formulación de un argumento. En el caso de Brown, los experimentos mentales habilitan la captación a priori de leyes naturales, lo que permite explicar que en ellos tenga lugar conocimiento nuevo sin la presentación de nuevos datos, Aunque consideramos que la perspectiva experimental es la más viable para proporcionar una explicación del funcionamiento de los experimentos mentales que tome en consideración estos rasgos, los desarrollos al respecto llevados a cabo por Brown asumen una serie de compromisos que la hacen poco factible. Presentaremos a continuación un análisis de los mismos.

\section{La captación intuitiva de leyes en los experimentos mentales platónicos}

En la explicación de los poderes epistémicos de los experimentos mentales, se entrecruzan dos cuestiones fundamentales: establecer cuál es el objeto de conocimiento científico y determinar a través de qué mecanismos accedemos a él. La perspectiva argumental traslada la respuesta general que el empirismo da a estas cuestiones: el conocimiento experimental nos vincula con instancias de las leyes que expresan regularidades en la naturaleza. Dentro de la perspectiva experimental, la de Brown manifiesta un compromiso con el platonismo: las leyes son relaciones entre universales que habitualmente conocemos inductivamente a través de la experiencia pero en el caso de los experimentos mentales, captamos intuitivamente. Examinaremos a continuación las razones que encuentra para suscribir estas tesis.

De acuerdo con Brown, el realismo es la mejor explicación del éxito y del progreso de la ciencia. En esta línea considera que el regularismo es una perspectiva insuficiente para distinguir leyes de generalizaciones accidentales y evitar el relativismo. Por lo que suscribe el realismo nomológico profesado en líneas generales por Dretske (1977), Tooley (1977) y Armstrong (1985) (de aquí en más, DTA). De acuerdo con Brown, las regularidades expresadas por una ley se sostienen en virtud de una relación irreductible entre universales, denominada relación de necesitación. La relación de necesitación o necesidad nómica es un primitivo postulado por razones explicativas. Es asimismo, contingente ya que la relación de necesitación entre universales es postulada solo para el mundo actual, otros mundos posibles podrían tener diferentes leyes para las que se sostienen relaciones diferentes. Además, esta relación entre 
universales puede implicar regularidades empíricas pero no está implicada por ellas: así es verdadero que $N(F, G) \vDash(\forall x)(F x \supset G x)$, pero no es verdadera la conversa $(\forall x)(F x \supset G x) \vDash N(F, G)$.

Para Brown la existencia de leyes no instanciadas en la física de partículas es una prueba de que debe admitirse la existencia independiente de relaciones entre universales (Brown, 1994, p. 98). Asimismo, el conocimiento de las leyes, entendidas como relaciones entre entidades abstractas, manifiesta la posibilidad del conocimiento humano de trascender la experiencia sensible. Brown admite que generalmente accedemos a las leyes por medio del conocimiento de sus instancias. Pero, en casos especiales, podemos evitar este paso y acceder directamente a los universales relacionados. Este es un añadido a su concepción metafísica de las leyes, que no comparten ni Dretske ni Tooley ni Armstrong. Para Brown si bien generalmente nuestro conocimiento de las leyes es inductivo, es posible admitir una forma de conocimiento independiente de la experiencia. Los experimentos mentales platónicos habilitan esta vía de conocimiento a priori.

La posibilidad de conocimiento a priori en física está dada para Brown al admitir la posibilidad del conocimiento a priori en matemática. Los objetos matemáticos existen fuera del espacio y del tiempo y pueden ser captados por la mente. Si se acepta la verdad del platonismo en matemática ${ }^{4}$ y se da por legítima su concepción de las leyes naturales, esto es que las leyes son universales relacionados de cierta manera, el conocimiento intuitivo de las leyes del mundo físico resulta altamente plausible.

La estrategia que emplea Brown para argumentar a favor de esta tesis consiste en asimilar la intuición racional al proceso de percepción visual. El proceso de percepción visual se explica apelando a la interacción de los fotones con los conos y bastones que se encuentran en la retina y la transferencia de la señal a través del nervio óptico hasta la corteza visual. De una manera análoga podemos explicar la captación intelectual de leyes naturales. Debido a que la explicación ampliamente aceptada del proceso de percepción visual deja sin explicar cómo un proceso físico causa una creencia, el mismo tipo de ignorancia es aceptable al explicar el caso de la captación de objetos matemáticos. Según Brown, el conocimiento intuitivo de verdades matemáticas explica la compulsión a creer que " $7+5=12$ ", de manera similar a como el empirismo explica la compulsión a creer que el césped es verde.

4 Los argumentos esgrimidos por Brown para defender el platonismo en matemática los desarrolla en su monografía dedicada enteramente a este tema (Brown, 2008). Una de las principales razones para sostener esta posición, es que, de acuerdo con Brown permite explicar la verdad de las proposiciones matemáticas. 
Brown admite que el conocimiento a priori también es posible en el ámbito de las ciencias naturales. Ciertos fenómenos, concebidos con independencia de la experiencia, pueden exhibir las propiedades de clase natural y posibilitar de ese modo la captación de leyes de la naturaleza. La noción de fenómeno ocupa un lugar de importancia en la justificación que Brown proporciona de la posibilidad del conocimiento a priori en los experimentos mentales platónicos. Los fenómenos son postulados como mediadores entre el mundo empírico y las leyes físicas. Brown sostiene que ciertos fenómenos pueden ser concebidos con independencia de los datos empíricos. Para solventar esta posición se refiere a la noción de fenómeno desarrollada por Bogen y Woodward (1988), ampliamente aceptada en el contexto de la filosofía de la experimentación, según la cual los datos son idiosincráticos y proveen evidencia del fenómeno. Desde esta perspectiva solo los datos son observables en sentido estricto. Los fenómenos, entendidos como formas estables y universales en las cuales el mundo se encuentra articulado, no son observables en ningún sentido interesante del término sino que son construidos a partir de los datos. Brown amplía estas conclusiones y sostiene que los fenómenos son entidades abstractas que corresponden a clases naturales: "Los científicos construyen fenómenos a partir de una gran masa de datos singularizando lo que toman como clases naturales genuinas" (Brown, 1994, p. 125).

La construcción del fenómeno es un proceso que Brown equipara con la dialéctica ascendente a la que Platón refiere en la metáfora empleada en Fedro 265e: "el carnicero es capaz de seccionar el cuerpo según sus articulaciones". El aspecto visualizable del fenómeno no entra en contradicción con su alteridad respecto de los datos empíricos y no implica que el fenómeno pueda ser percibido. Los fenómenos que son captados como imágenes en los experimentos mentales platónicos son ejemplares de clases naturales. Tienen relación con la observación dado su carácter visual, pero deben distinguirse de los datos de la percepción sensible porque son objetos abstractos. Es posible intuir leyes de la naturaleza en estas situaciones de visualización precisamente porque los fenómenos ejemplifican clases naturales o patrones. Las clases naturales corresponden a un tipo de propiedad esencial que implica que si un miembro de la clase tiene esa propiedad, entonces, todos los miembros la tienen. Los experimentos mentales platónicos exhiben este tipo de fenómenos.

La tesis de Brown según la cual los experimentos mentales proporcionan conocimiento a priori se funda en una metafísica robusta acerca de la existencia de entidades abstractas, los universales, de las leyes naturales como relaciones entre universales, instanciados o no, de clases naturales, y de fenómenos independientes de los datos de la percepción sensible. El análisis de los experimentos mentales 
de Brown no parece proporcionar razones adicionales a las que han ofrecido los metafísicos para adoptar esta perspectiva. La postulación de fenómenos y de la captación intuitiva de leyes no parece explicar suficientemente el caso del experimento de los cuerpos en caída libre. La visualización de objetos de distinto peso y del sistema de objetos compuestos en caída libre parece cumplir una función al ilustrar las consecuencias absurdas de la explicación aristotélica, pero por sí mismo no proporciona evidencia para la hipótesis de que la caída es una constante que no depende de ninguna de las variables relevantes.

La posición empirista, por el contrario, provee una explicación más simple que hace superfluos a los compromisos metafísicos a los que está ligado el platonismo de Brown. No obstante la perspectiva experimental defendida por Brown explica algunos rasgos importantes de los experimentos mentales. La visualización de un escenario y la realización de alguna actividad en la imaginación son aspectos esenciales del funcionamiento de estos dispositivos. Si bien la descripción del escenario y las instrucciones son comunicadas a través de una narrativa, el resultado del experimento se obtiene razonando sobre la situación imaginada pero no es el producto de una inferencia inductiva o deductiva. El hecho de que los experimentos mentales puedan ser reconstruidos como argumentos no constituye una prueba de que estos sean esencialmente inferencias. No obstante, aceptar que los experimentos mentales son eventos o procesos, y con ello, intentar proveer una explicación de su funcionamiento desde la perspectiva experimental, no nos compromete necesariamente con los supuestos metafísicos asumidos por Brown. Intentaremos mostrar a continuación dos alternativas para dar cuenta del tipo y el objeto de conocimiento de los experimentos mentales que Brown llama "platónicos" que ni los reducen a inferencias inductivas o deductivas ni se comprometen con el regularismo respecto de las leyes.

\section{Experimentos mentales. Realismo sin justificación a priori}

La perspectiva argumental constituye un marco austero para dar cuenta del valor epistémico de los experimentos mentales en términos de nociones corrientes de la lógica. Se trata, en definitiva, de mostrar que bajo la apariencia de un experimento (cf. Norton, 1996, p. 334) -construida por medio de la descripción de un escenario, la referencia a instrucciones y una narrativa en términos de procesos y resultados- se esconde en realidad tan solo un argumento deductivo o inductivo:

Los experimentos mentales son dispositivos que reorganizan o generalizan lo que ya sabemos acerca del mundo y lo hacen explícito. [...] En tanto dispositivos que 
meramente reorganizan (información) son argumentos deductivos. En tanto generalizan, en sentido amplio, son argumentos inductivos. El resultado es confiable solo en tanto, nuestras suposiciones son verdaderas y la inferencia es válida (Norton, 1996, p. 336).

El costo inmediato que la perspectiva argumental ha de estar dispuesto a pagar consiste, como hemos visto, precisamente en la negación del carácter genuinamente experimental de los experimentos mentales. Dicho carácter no solo se ancla a la convención de llamarlos o concebirlos de cierto modo, sino (al menos) a dos razones de peso. Primero, buena parte de los experimentos mentales efectivamente empleados en ciencia resultan intuitivamente experimentos, y no meros argumentos coloridos, o ilustrados imaginativamente. Segundo, varios de ellos fueron considerados decisivos para el abandono de ciertas teorías y la aceptación de otras, función que no parece poder ser cumplida por la mera reorganización deductiva de información ya conocida. Con todo, estas no son sino las consecuencias obvias de negar el carácter experimental de estos dispositivos de conocimiento. Como hemos visto, la tesis de que los experimentos mentales son reducibles a meros argumentos parece resignar, además, tres aspectos centrales a su función y estatus epistémico: su valor heurístico, su capacidad de producir conocimiento nuevo y su valor evidencial, fundamental en la elección de teorías.

Por su parte, la perspectiva argumental cuenta con recursos suficientes para dar cuenta de dichos aspectos y salvar el carácter sustantivo y auténticamente experimental de los experimentos mentales. Sin embargo, dichos recursos requieren asumir compromisos metafísicos que muchos (no solo los defensores de la perspectiva argumental) podrían juzgar inflacionarios. Esos compromisos se despliegan en dos frentes complementarios: el tipo de conocimiento que se atribuye a los experimentos mentales, y el objeto de conocimiento que se postula para ellos. Como hemos visto, para la perspectiva argumental la realización de un experimento mental involucra la captación, mediante una intuición intelectual, de una fundamentación a priori de cierta regularidad del mundo natural. Esa fundamentación, ese objeto de conocimiento para el experimento mental, no es otra cosa que una ley de la naturaleza entendida al modo de la DTA, i.e., como una relación de necesitación entre universales de primer orden.

Claramente, estos compromisos son evitados por la perspectiva argumental, pero su visión deflacionaria entra en tensión con algunas intuiciones básicas acerca de los experimentos mentales. En la presente sección mostraremos cómo es posible arribar a una solución intermedia que permite salvar las ventajas de la perspectiva argumental sin abrazar una metafísica inflacionaria respecto del tipo de conocimiento involucrado en los experimentos mentales. En la sección 
siguiente haremos lo propio respecto del objeto de conocimiento que se les atribuye.

Creemos que posible aceptar la tesis de la perspectiva experimental según la cual lo que ocurre en un experimento mental no es la mera reorganización del conocimiento en un argumento, sin por ello comprometerse con una posición apriorista. Si bien la observación y el rol activo del experimentador son fundamentales para la formación de creencias acerca del mundo natural en los experimentos mentales, esto no implica aceptar que las nuevas creencias que nos formamos como producto de este proceso se encuentran justificadas $a$ priori. Concretamente, sostenemos que lo que tiene lugar en un experimento mental es una inferencia a la mejor explicación (IME) en la que el fenómeno que resulta de la realización del experimento mental se juzga fundamentado en una base metafísica. En la sección siguiente veremos con detalle en qué consiste ese fundamento metafísico, y cómo éste puede ser diferente de leyes de la naturaleza concebidas al modo de la DTA. Quedan entonces dos cuestiones principales por atender: precisar cómo es que la IME se aplica en el contexto de los experimentos mentales, y dar cuenta de cómo dicho mecanismo inferencial es capaz de recuperar todas las virtudes del enfoque experimental. Si tenemos éxito, habremos elaborado una posición que, pese a caracterizar los experimentos mentales como procesos que involucran más de un tipo de inferencia (deductivas y a la mejor explicación), reivindica el genuino carácter experimental de estos dispositivos de conocimiento y, por tanto, se enmarca en lo que hemos denominado perspectiva experimental. Dicho carácter queda anclado, como veremos, al aspecto creativo (también llamado paso abductivo) de la IME, cuya naturaleza no parece reducible a un mero paso inferencial.

Es preciso entonces, antes de comenzar nuestra argumentación, dar una breve mirada a la naturaleza de ese mecanismo. La IME es una forma de razonamiento ampliativo presentada por Harman (1965) como un procedimiento similar a lo que Peirce (1958) denominó abducción. Consiste en inferir una cierta hipótesis a partir de la premisa que afirma que ella brinda una mejor explicación potencial de cierta evidencia dada que ninguna otra hipótesis disponible. De ese modo, la IME pone en relación el valor explicativo de una hipótesis con su valor epistémico. El mismo Peirce (1958, p. 2623) y muchos influyentes teóricos de la IME, Michalski (1987, p. 188) y Psillos (2000, p. 60), han afirmado que se trata de un tipo de razonamiento ampliativo y, por lo tanto, distinto de la deducción, pero también diferente de la inducción. Todos ellos han señalado la importancia de esta forma de inferencia para la práctica científica, tanto en el descubrimiento como en la confirmación de hipótesis. Han subrayado, además, la importancia de su rol tanto en ciencia aplicada, como en la investigación 
básica. Del mismo modo, la IME es central al debate filosófico acerca de la ciencia, en tanto el principal argumento a favor del realismo científico, el llamado argumento del "no-milagro", toma la forma de una IME (cf. Putnam, 1975, Psillos, 1999 y Chakravartty, 2007).

La IME, al igual que la inducción, se encuadra dentro de los razonamientos ampliativos. En tal sentido, la conclusión contiene más información que las premisas y, por ende, su verdad no está garantizada. Sin embargo, es preciso destacar que difiere de una generalización inductiva en el modo en que dicha ampliación tiene lugar. Como es sabido, una generalización inductiva procede proyectando la extensión de una propiedad a todo un conjunto, sobre la base de que algunos miembros de ese conjunto instancian la propiedad en cuestión. La IME, en cambio, involucra un momento creativo, un salto abductivo en el que una hipótesis aparece como una buena explicación de cierta evidencia disponible. Este proceso no es algorítmico, es por ello que para Pierce "la sugerencia abductiva viene a nosotros como un destello [...] es un acto de intuición [insight]" (Peirce, 1958, p. 5181), por el contrario, "la inducción es un proceso para testear hipótesis que ya están disponibles. La inducción no añade nada" (Peirce, 1958, p. 7217). Este aspecto creativo asociado a la generación la(s) nueva(s) hipótesis explicativas que será(n) objeto de selección no es formalizable ni pasible de un tratamiento en lógica estándar.5 Además, existe un elemento adicional para justificar dicha diferencia. Las inducciones, concebidas al modo tradicional, pueden ser generalizaciones (enumerativas) o razonamientos analógicos. Pero existe otra forma de salto inferencial que se distingue de los anteriores, a saber, aquel que va desde la regularidad expresada en un enunciado general a la necesidad subyacente a esa regularidad, en otras palabras, el salto de generalizaciones a leyes. No obstante, consideramos que es un error identificar este tipo inferencia con la inducción. Exponer nuestras razones nos alejaría del foco de estas páginas, pero baste decir que la proyección de una regularidad desde lo observado a lo observable no es suficiente para establecer una conexión necesaria de re entre conjunciones constantes. Por el contrario, se requiere para eso un tipo de explicación que, en la literatura reciente, se suele denominar explicación metafísica o fundamentación (grounding). De ese modo, el salto de la regularidad a la necesidad no puede ser meramente

5 Es cierto que en sistemas multiagente (sistemas multimodales, con operadores de conocimiento y creencia), el problema de la selección de abducibles no queda, por así decir, al margen del tratamiento lógico del proceso abductivo (cf. Nepomuceno-Fernández et al., 2014). No obstante, consideramos que la necesidad de introducir operadores epistémicos en un sistema para dar cuenta de los fenómenos ligados a la IME muestra claramente que las dimensiones en que este mecanismo inferencial tiene lugar (epistémica, cognitiva, pragmática) escapan a las capacidades expresivas de sistemas deductivos o inductivos clásicos. 
inductivo. Sostenemos que esa noción de explicación es la que subyace a la IME en el caso de los experimentos metales. Volveremos sobre ese punto luego.

Como ya hemos adelantado, nuestra tesis central es que lo que realmente acontece en la realización de un experimento mental no es la captación a priori de una ley natural, sino una IME. No negamos, al igual que Brown no lo hace, que parte de los experimentos mentales consiste en una inferencia deductiva o inductiva. La parte destructiva del experimento de Galileo es, sin duda alguna, una reductio. Sin embargo, como hemos señalado, la pieza de conocimiento nuevo que dicho experimento aporta, a saber, el principio de equivalencia, no puede derivarse de las premisas inductivamente y solo se sigue deductivamente de ellas de un modo trivial. Es por ello que, según Brown, es necesario suponer que la captación intuitiva de leyes dota a experimentos mentales de poderes epistémicos extraordinarios que les permite, simultáneamente, refutar una teoría y proporcionar evidencia para una nueva. En el experimento de Galileo experimentador se representa lanzando objetos desde una torre en un medio sin fricción. En este contexto es imposible imaginar un objeto compuesto de cuerpos de distinto peso que caiga de acuerdo al principio aristotélico. Según Norton, el experimento puede reconstruirse como una reducción al absurdo del principio aristotélico de acuerdo con el cual, la velocidad de caída de los cuerpos es directamente proporcional a su peso. El problema con esta interpretación es, para Brown, que no explica cómo es posible pasar de esta inferencia a la formulación del principio de equivalencia, dado que cualquier cosa puede seguirse de una contradicción. Para Brown el tipo de fenómeno que nos representamos en la imaginación provee, por sí mismo evidencia para la aceptación de una nueva teoría. Tiene lugar en este caso una forma de conocimiento no inferencial que permite a los experimentadores trascender la experiencia y contemplar directamente la ley que explica el fenómeno.

Sin embargo, sostenemos, no es preciso ir tan lejos en la especulación metafísica, ni resignarse a la reducción del aporte del experimento a una inferencia deductiva o inductiva. La IME puede constituirse como un terreno intermedio para caracterizar el tipo de conocimiento que aportan los experimentos mentales. En el caso de Galileo, por ejemplo, el salto hacia la proposición que sostiene que la velocidad de la caída es una constante, y no depende del peso y la composición de los cuerpos no puede darse inductivamente. Deductivamente, en cambio, cualquier proposición puede ser inferida. Pero, ¿por qué inferir esa en particular? Afirmamos que el principio de equivalencia constituye la hipótesis que mejor explica tanto la falsedad de los principios aristotélicos destruidos por la reductio como los fenómenos visualizados en el experimento.

Expuesta nuestra tesis sobre el rol de la IME en los experimentos mentales, resta mostrar cómo es capaz de recuperar todas las virtudes del enfoque 
experimental. El valor heurístico de los experimentos mentales tal vez sea el rasgo que más fácilmente puede ser recogido en el marco de la IME. En líneas generales existen dos miradas acerca del modo en que una IME procede. Para algunos (e.g. Josephson, 2000, Psillos, 2000) la abducción es un proceso simple en el que una única hipótesis es construida como la mejor explicación de cierto fenómeno. Para otros (e.g. Aliseda, 2000, Denecker et at., 1996, Bessant, 2000 y Flach, 2000), en cambio, se trata de un proceso de dos pasos: uno productivo, en el que se crea un lote de hipótesis que constituyen explicaciones potenciales de la evidencia, y uno selectivo, en el que se infiere la que se considera la mejor de las disponibles. En cualquier caso, es universalmente reconocido que la IME juega un papel relevante orientando la investigación hacia la generación de nuevas hipótesis científicas. En ese sentido en que Schupbach afirma que en el marco de la investigación científica la IME resulta "útil como una heurística informal que nos permite aproximarnos razonamientos probabilísticos sólidos" (2016, p. 8). El mismo Pierce, de hecho, articula esta idea en su teoría inferencial tardía, que tiene a la abducción, la deducción y la inducción como las tres etapas de la investigación científica, y donde la primera concierne a la generación de una hipótesis capaz de explicar adecuadamente las observaciones.

No muy lejos de estas intuiciones está la cuestión la capacidad de producir conocimiento nuevo. El valor heurístico del IME y su rol en la investigación de nuevas hipótesis podría considerarse prueba de dicha capacidad. Del mismo modo, el hecho de que la IME constituye una forma de razonamiento ampliativo basta, al menos en un sentido amplio, para asegurar que la conclusión nos ofrece un plus de conocimiento, no contenido en las premisas. Sin embargo, podría argüirse que ello no resulta suficiente para dar cuenta del tipo de conocimiento nuevo que un experimento mental parece producir. Después de todo, como hemos visto, el mismo Norton sostiene desde la perspectiva argumental que los experimentos mentales pueden reducirse tanto a razonamientos deductivos como inductivos, siendo estos últimos ampliativos. El problema entonces no es el aumento de conocimiento empírico, sino la naturaleza de ese conocimiento:

El elemento esencial en la experimentación es el mundo natural. [...] El solo hecho de imaginar lo que el mundo podría hacer si lo manipuláramos de esta u otra manera parecería ser inútil, ya que omite este elemento esencial. Sin embargo, la literatura científica frecuentemente nos conduce a tales experimentos imaginarios, realizados puramente en la mente, y con considerable beneficios aparentes. [...] Si esto pudiese tomarse literalmente, los experimentos mentales realizan magia epistémica. Nos permiten usar el pensamiento puro para descubrir cómo es el mundo. $\mathrm{O}$ al menos se trata de una magia dudosa para un empirista que cree que solo podemos descubrir cómo es el mundo a partir de nuestra experiencia del mundo (Norton, 2004a, p. 45). 
Una lectura de primera mano podría imputar cierta ingenuidad filosófica al diagnóstico de Norton. Ya Hempel (1966) dió cuenta de la (en su opinión) imposible tarea de dar con una lógica del descubrimiento. Parte de su argumento consiste en señalar que las hipótesis teóricas de alto poder explicativo no pueden derivarse inductivamente de los datos, solo accedemos a ellas mediante el ejercicio de la creatividad. En cualquier caso, esa "magia epistémica" que los experimentos mentales parecen aportar consiste en la aprehensión de alguna pieza de información que (en algún sentido interesante de "derivar") no podría derivarse de la evidencia disponible. No es difícil ver que buena parte del problema consiste en especificar adecuadamente dicho sentido. Conocemos la opción de Brown: dado que ese conocimiento nuevo no puede obtenerse ni inductiva ni deductivamente a partir de la experiencia, debemos aceptar que su fuente es a priori y no inferencial, más precisamente, la captación intuitiva de leyes de la naturaleza. El rechazo de esta jugada por parte de la perspectiva argumental no ha de llamarnos la atención, pero sí el hecho de que se funda en un supuesto común: la imposibilidad de obtener conocimiento nuevo (en el sentido especificado previamente) acerca del mundo natural por medios deductivos o inductivos. En el marco de una postura empirista, tal supuesto conduce inevitablemente a ubicar la metafísica de Brown en el terreno de la superstición.

Nuestra propuesta consiste en señalar que abrazar dicho supuesto no tiene por qué implicar que el conocimiento nuevo aportado por los experimentos mentales sea a priori y no inferencial. La IME es un tipo de inferencia capaz de dar cuenta del salto creativo descrito por Hempel sin necesidad de "magia epistémica" alguna. Es en tal sentido que Flach (2000) caracteriza la IME como la lógica de la generación de hipótesis. Del mismo modo, Hanson (1960) propuso que la abducción de Peirce da razones para sugerir una hipótesis, más bien que para aceptar una hipótesis, y para Levi (1995, p. 85), la tarea de la abducción es establecer la "partición final [ultimate partition]" de respuestas potenciales a un problema cognitivo.

Por último, resta la cuestión del valor evidencial atribuido a los experimentos mentales. Una primera ruta para dar cuenta de este aspecto por vía de la IME requiere poner el foco en su rol en el contexto de justificación. No todos comparten la opinión de Hanson respecto de que la IME corresponde únicamente al contexto de descubrimiento. Niiniluoto (1999, 2004), Flach (2000) y Schupbach (2016), entre otros, han señalado que puede confirmarse/probabilificarse una hipótesis en razón de su poder explicativo. En la misma línea, Dragulinescu (2016) sostiene que la IME puede formularse como teoría acerca del valor de la evidencia. En segundo lugar, debe atenderse también a las implicaciones precisas de su 
fase selectiva. La inferencia de una hipótesis en virtud de su poder explicativo conlleva la eliminación de las hipótesis alternativas. Este rasgo permite asignar a la IME un rol en la elección entre teorías rivales-de hecho, según Kuipers (2004), la centralidad de dicha función amerita que se la reconceptualice como una Inferencia a la Mejor Teoría. Como hemos visto, la principal razón para asignar valor evidencial a los experimentos mentales reside en su papel en la elección de teorías rivales.

En resumen, una concepción sustantiva de los experimentos mentales capaz de dar cuenta de sus ventajas intuitivas (valor heurístico, capacidad de producir conocimiento nuevo y valor evidencia) no requiere de la postulación mecanismo de conocimiento a priori por medio de intuiciones platónicas.

\section{Necesidad natural sin Realismo Nomológico}

Renunciar al apriorismo en el marco de una concepción sustantiva acerca de los experimentos mentales es sin dudas un signo de aproximación a la austeridad metafísica que caracteriza la perspectiva argumental. Sin embargo, esa descripción del tipo de conocimiento no sería del todo significativa si aceptara que el objeto de conocimiento de los experimentos mentales son leyes de la naturaleza, es decir, el mismo postulado por la versión más radical de la perspectiva experimental. Hemos visto que para Brown lo que se conoce en la realización de un experimento mental es una ley de la naturaleza, entendida al modo de del realismo nomológico de DTA, a saber, como un universal platónico de segundo orden que se instancia en universales de orden inferior. La alternativa natural a esta metafísica -abrazada por los empiristas como Norton-, es la teoría regularista de las leyes (o más brevemente, el regularismo). El regularismo, en su lugar, adopta una metafísica humeana que rechaza todo tipo de necesidad de re. Según la perspectiva argumental, el resultado de un experimento mental no es otra cosa que la reorganización de cierta información conocida acerca de las regularidades naturales que hemos ganado por medio de la experiencia (o su mera proyección inductiva), de ese modo, los objetos de conocimiento de dichos “experimentos" no son otra cosa que las regularidades empíricamente accesibles. Ya hemos señalado que este recurso resulta explicativamente insuficiente. La información conocida sobre regularidad no es capaz de dar cuenta de varias intuiciones básicas acerca de los experimentos mentales, fundamentalmente, aquella según la cual brindan conocimiento nuevo acerca del mundo natural. Además, incluso la mera proyección inductiva de regularidades conocidas queda carente de todo sustento en este marco. 
Nuestra opción, por tanto, no será ni el regularismo empirista ni el realismo nomológico platonista de la perspectiva experimental. Sostenemos que es posible admitir una forma de necesitarismo más débil que el realismo nomológico de la DTA, sin caer en el humeanismo de los regularistas: los experimentos mentales (cuyo mecanismo de producción de conocimiento es la IME) tienen como objeto de conocimiento la estructura disposicional del mundo natural. A fin de poner carne en los huesos de esta propuesta, es preciso revisar con cierto detalle la polémica acerca del estatus metafísico de las leyes naturales.

Para entender apropiadamente la variedad de posiciones enfrentadas, dos importantes distinciones deben ser trazadas entre ellas. La primera diferencia a los regularistas de los necesitaristas, la segunda a los realistas de los antirrealistas nomológicos.

El regularismo se enmarca en la tradición empirista que reivindica cierto escepticismo respecto de los compromisos metafísicos. Con todo, la inspiración humeana no es suficiente para arrojarlo a un escepticismo radical. La metafísica humeana del regularismo es, después de todo, una propuesta metafísica. Para quienes sostienen posiciones en esta línea el mundo está constituido por eventos discretos, cada uno de los cuales se autocontiene y es tanto ontológica como modalmente independiente del resto, una suerte de mosaico cuyas piezas son estos eventos discretos y causalmente inertes. Muchas de esas piezas se presentan en relaciones regulares de semejanza y contigüidad espaciotemporal, pero fuera de ellas ninguna relación de necesidad las conecta. A decir de Lewis, solo eso es nuestro mundo, una cosita y luego otra (1986, p. ix). La analogía es pertinente en un aspecto más: la independencia de cada una de las piezas no implica que no haya regularidades; por el contrario, la investigación empírica puede encontrar complejos patrones en ese mosaico, sin embargo, ninguno de esos patrones está determinado por algún tipo de necesidad, y por lo tanto no son proyectables más allá de lo observado.

El necesitarismo, por su parte, afirma la existencia de conexiones necesarias en la naturaleza. Sin embargo, no debe ser identificado con el realismo acerca de las leyes naturales: todo realismo nomológico es necesitarista, pero no todo necesitarismo debe afirmar la existencia de leyes. El fin de adoptar el necesitarismo es, en este contexto, brindar un relato explicativo acerca del fundamento de las regularidades naturales, a diferencia del regularismo, para el que esa regularidad no está fundamentada y por tanto no requiere explicación. Muchas han sido las variaciones de ese relato, universales, esencias, poderes y disposiciones fueron invocados como el soporte ontológico de los patrones naturales que escapan a la contingencia. Los argumentos en este sentido han seguido una estructura relativamente constante, aduciendo que todo un conjunto 
de rasgos de nuestro mundo que remiten a la regularidad (la posibilidad de realizar inducciones, de sostener enunciados contrafácticos, de realizar predicciones e incluso de la actividad científica como tal) quedaría inexplicado si no se presumiera la existencia de un fundamento para la necesidad natural.

Esto nos permite abordar la segunda distinción relevante en el debate. Solo aquellos necesitaristas para quienes el fundamento de la regularidad sean las leyes defienden un realismo nomológico. Su forma más popular es la DTA: las leyes son un tipo de universal de segundo orden que se instancia en universales de orden inferior. Dichos universales de primer orden mantienen relaciones de necesitación, esas relaciones de necesitación, instanciadas sobre estados de cosas, son las leyes. Si tener la propiedad $F$ implica tener la propiedad $G$, eso se debe a que $F$ y $G$ están vinculados en una relación $N$ de necesidad tal que $N(F, G)$. Este, sin embargo, no es el único relato disponible. Para la concepción internalista de Ellis (2001) las leyes existen, pero son internas a sus instancias; más precisamente, son las propiedades esenciales de las clases naturales.

Teniendo en cuenta ambas concepciones, Mumford (2004, p. 67) propone dos requisitos básicos que una teoría acerca de las leyes debe cumplir a fin de enrolarse en el realismo nomológico:

(a) Las leyes deben ser entendidas como una "adición de ser".

(b) Las leyes deben gobernar sus instancias.

La primera implica que cualquiera que decida ser realista acerca de las leyes debe comprometerse con el hecho de que éstas tienen algún tipo de espesor ontológico, deben constituir una categoría ontológica per se. El segundo supuesto implica que el rol de gobierno sobre sus instancias es no solo es una característica constitutiva de las leyes mismas, sino un requisito para la viabilidad de una metafísica que las postule.

El punto central de este recorrido por el debate en torno a las leyes es que la adopción de compromisos modales no es condición suficiente para suscribir el realismo nomológico, es decir, ser necesitarista no implica asumir que existen cosas tales como leyes en la naturaleza. El antirrealismo nomológico, por tanto, incluye todo el arco de posiciones regularistas y aquellas necesitaristas para las que las conexiones necesarias en la naturaleza están fundadas en alguna cosa diferente a las leyes. La adhesión a esta última forma de antirrealismo nomológico suele incluir la adopción de una metafísica disposicionalista: los poderes y disposiciones dotan al mundo de la efectividad causal que se requiere para fundamentar ontológicamente las regularidades. De ese modo, los compromisos modales se dirigen a los poderes y disposiciones inmanentes 
a las propiedades que constituyen el mundo natural. Posiciones en esta línea han sido defendidas por Mumford (2004) y Cartwright (1989, 1999, 2003). Alguien podría argüir que un compromiso tal es equiparable al del realismo nomológico, sin embargo, ha de reparase que la propuesta disposicionalista no cumple los requisitos (a)-(b), en tanto los poderes y disposiciones no representan ninguna adición de ser ni gobiernan a sus instancias, sino que son internos y autogobernantes (cf. Mumford, 2004, p. 194).

En la sección precedente afirmamos que, en cuanto al tipo de conocimiento aportado por los experimentos mentales, adoptando el marco de la IME es posible renunciar tanto al apriorismo implícito en la intuición intelectual de leyes, como a la mera reorganización argumental de información conocida. En cuanto al objeto de conocimiento alcanzado en esa inferencia, creemos que es posible encontrar un punto medio entre los universales platónicos de la DTA y el regularismo empirista implícito en la perspectiva argumental. Concretamente, sostenemos que los experimentos mentales permiten capturar la estructura disposicional de las entidades y procesos involucrados. El rédito epistémico de este compromiso es equivalente al de adoptar el realismo nomológico: el conocimiento alcanzado en un experimento mental trasciende la mera reorganización de información empírica, por el contrario, está fundamentado en rasgos modales del mundo. No es otra cosa que el conocimiento de ciertas conexiones necesarias entre propiedades naturales. Es este conocimiento el que garantiza la proyección inductiva de las regularidades empíricas. Lo único que se resigna en esta jugada es el carácter a priori de ese conocimiento, y con él, su infalibilidad. Con todo, consideramos que ninguno de esos aspectos constituye un valor por sí mismo, ni siquiera en las más robustas concepciones realistas acerca de la ciencia, donde el falibilismo y el naturalismo son más bien la regla que la excepción. Por otra parte, la inversión metafísica es claramente menor que en el caso de la perspectiva experimental de Brown. La aprehensión de ciertas relaciones modales objetivas del mundo natural no requiere de la postulación de universales de orden superior ni de un mecanismo de captación intuitiva de ellos.

Si bien alejada de la discusión acerca de los experimentos mentales, la posición de Dragulinescu puede tomarse como próxima al tipo de estrategia que proponemos. Según Dragulinescu (2016, p. 222), la IME permite la generación de hipótesis mecanicistas en el marco de investigaciones en medicina y la evaluación de evidencia a favor de la existencia de dichos mecanismos. La identificación de mecanismos causales, del mismo modo, no está lejos de los principios del disposicionalismo que defendemos. De hecho, Cartwright (1989) afirma que las disposiciones y capacidades constituyen el marco metafísico más indicado para dar cuenta de las explicaciones mecanicistas en medicina y biología. 


\section{Conclusión}

A lo largo del presente trabajo hemos presentado una nueva concepción acerca de los experimentos mentales que procura salvar lo mejor de dos mundos: por una parte, preserva el poder explicativo la perspectiva experimental, que hace posible reivindicar y fundamentar nuestras intuiciones más básicas acerca de la naturaleza, función y estatus epistémico de los experimentos mentales; por otra parte, retiene al menos parte del espíritu de austeridad metafísica que motiva el relato de la perspectiva argumental.

En primer lugar, hemos sostenido que el mecanismo epistémico que opera en los experimentos mentales es una IME. De ese modo, es posible dar cuenta de los rasgos centrales que la perspectiva experimental atribuye a los experimentos mentales: valor heurístico, capacidad de producir conocimiento nuevo y valor evidencial. Sin embargo, nuestra propuesta no necesita postular intuiciones a priori para dar cuenta de su poder epistémico. En segundo término, afirmamos que para dar cuenta de dichos rasgos no es preciso postular leyes naturales como el objeto de conocimiento de los experimentos mentales. Una fundamentación metafísica igualmente sólida de sus capacidades epistémicas se obtiene postulando una estructura disposicional en la naturaleza. Ni el apriorismo ni el realismo nomológico son necesarios para explicar las particularidades de estos dispositivos de conocimiento científico, una metafísica más modesta puede hacer el trabajo. En suma, nuestra propuesta obtiene los mismos réditos epistémicos con menor inversión metafísica.

\section{Referencias}

ALISEDA, A. "Abduction as epistemic change: A Peircean model in artificial intelligence”. (pp. 45-58). In: Abduction and Induction. Springer Netherlands, 2000. ARMSTRONG, D. M. "What is a Law of Nature?". Cambridge University Press, 1985. BESSANT, B. "On relationships between induction and abduction: a logical point of view". (pp. 77-87). In: Abduction and Induction. Springer Netherlands, 2000.

BOGEN, J., WOODWARD, J. "Saving the Phenomena". The Philosophical Review, 97, pp. 303-352, 1988.

BROWN, J. R. "The Laboratory of the Mind: Thought Experiments in the Natural Sciences". London: Routledge, 1991.

. "Why Empiricism Won't Work". Proceedings of the Biennial Meeting of the Philosophy of Science Association, 1992. Volume Two, pp. 271-279.

1994.

"Smoke and Mirrors: How Science Reflects Reality". New York: Routledge,

"Why Thought Experiments Transcend Empiricism". (pp. 23-43). In: Hitchcock, C. (ed.). Contemporary Debates in the Philosophy of Science. Oxford: Blackwell, 2004a. 
. "Peeking into Plato's Heaven". Philosophy of Science, 71, pp. 1126-1138, $2004 \mathrm{~b}$. "Philosophy of Mathematics: A Contemporary Introduction to the World of Proofs and Picture". Second Edition. New York: Routlege, 2008.

"The Laboratory of the Mind: Thought Experiments in the Natural Sciences". Second Edition. London: Routledge, 2011.

BUZZONI, M. "Empirical Thought Experiments: A Transcendental-Operational View". Epistemologia. An Italian Journal for the Philosophy of Science, 33, pp. 05-26, 2010. CARTWRIGHT, N. "Nature's capacities and their measurement". Clarendon Press: Oxford, 1989.

. "The dappled world: A study of the boundaries of science". Cambridge University Press: Cambridge, 1999.

. "What is wrong with Bayes nets?". The Monist, Vol. 84, Nr. 2, pp. 242-264, 2001. ."What makes a capacity a disposition? Causality: Metaphysics and methods discussion paper 10/03". London School of Economics Centre for Philosophy of Natural and Social Science, 2003.

CHAKRAVARTTY, A. "A metaphysics for scientific realism: Knowing the unobservable". Cambridge: Cambridge University Press, 2007.

DENECKER, M., MARTENS, B., DE RAEDT, L. On the difference between abduction and induction: a model theoretic perspective. (pp. 19-22). In: Flach and Kakas, 1996. Available on-line at http: 1 lwww . cs. bris. ac. ukl-flachl ECAI961.

DRAGULINESCU, S. "Inference to the best explanation and mechanisms in medicine". Theoretical Medicine and Bioethics, Vol. 37, Nr 3, pp. 211-232, 2016.

DRETSKE, F. "Laws of Nature". Philosophy of Science, 44, pp. 248-268, 1977.

ELLIS, B. "Scientific essentialism". Cambridge University Press, 2001.

FLACH, P. A. "On the logic of hypothesis generation". (pp. 89-106). In: Abduction and induction Springer Netherlands, 2000.

GALILEO. (1638). "Discorsi e dimostrazione matematiche intorno a due nuove science". Leiden: Elsevier. [Traducción inglesa de Henry Crew and Alfonso de Salvio. New York: Dover, 1954.]

HANSON, N. R. "Is there a logic of scientific discovery?". Australasian Journal of Philosophy, Vol. 38, Nr. 2, pp. 91-106, 1960.

HARMAN, G. "The inference to the best explanation". Philosophical Review, 74, pp. 88-95, 1965.

HEMPEL, C. G. "Philosophy of Natural Science. Englewood Cliffs". NJ: Prentice Hall, 1966.

IRVINE, A. "Though Experiments in Scientific Reasoning". In: T. Horowitz y G. Massey (eds.). Thought Experiments in Science and Philosophy. Savage: Rowman and Littlefield, 1996.

JOSEPHSON, J. R. "Smart inductive generalizations are abductions". (pp. 31-44). In: Abduction and induction. Springer Netherlands, 2000.

KUIPERS, T. A. Inference to the best theory, rather than inference to the best explanationkinds of abduction and induction. (pp. 25-51). In: Induction and deduction in the sciences. Springer Netherlands, 2004. 
LEVI, I. "Induction According to Peirce". (pp. 59-93). In: K. L. Ketner (ed.). Peirce and Contemporary Thought. New York: Fordham University Press, 1995.

LEWIS, D. "How to define theoretical terms". The Journal of Philosophy, Vol. 67, Nr. 13, pp. 427-446, 1970.

. Philosophical Papers II, Oxford: Oxford University Press, 1986.

MICHALSKI, R. S. “Concept learning”. (pp. 185-194). In: S. Shapiro (ed.). Encyclopedia of Artificial Intelligence. John Wiley, Chicester, 1987.

MUMFORD, S. "Laws in nature". Routledge, 2004.

NEPOMUCENO-FERNÁNDEZ, Á., SOLER-TOSCANO, F., VELÁZQUEZQUESADA, F. R. "The Fundamental Problem of Contemporary Epistemology". Teorema: Revista Internacional de Filosofia, pp. 89-103, 2014.

NIINILUOTO, I. "Defending abduction". Philosophy of Science, 66, S436-S451, 1999. . "Truth-seeking by abduction". (pp. 57-82). In: Induction and deduction in the sciences. Springer Netherlands, 2004.

NORTON, J. “Thought Experiments in Einstein's Work”. (pp. 129-148). In: T. Horowitz, G. Massey (eds.). Though Experiments in Science and Philosophy. Savage, MD: Roward \& Littefield, 1991.

. “Are Thought Experiments Just What You Thought?”. Canadian Journal of Philosophy, 26, pp. 333-366, 1996.

"Why Thought Experiments Do Not Transcend Empiricism". (pp. 44-66).

In: C. Hitchcock (ed.). Contemporary Debates in the Philosophy of Science. Oxford: Blackwell, 2004a.

. "On Thought Experiments: Is There More to the Argument?". Philosophy of Science, 71, pp. 1139-1151, 2004b.

PEIRCE, C. S. "Collected Papers of Charles Sanders Peirce". Harvard University Press, Cambridge, Massachusetts. Edited by C. Harstshome, P. Weiss \& A. Burks, 1958.

PSILLOS, S. “Scientific Realism: How Science Tracks Truth”. London: Routledge, 1999. . "Abduction: Between conceptual richness and computational complexity". (pp. 59-74). In: Abduction and induction. Springer Netherlands, 2000.

PUTNAM, H. "Mathematics, Matter and Method". Cambridge: Cambridge University Press, 1975.

SCHUPBACH, J. N. "Inference to the best explanation, cleaned up and made respectable". In: Best Explanations: New Essays on Inference to the Best Explanation, 2016.

SORENSEN, R. "Thought Experiments". New York: Oxford University Press, 1992. TOOLEY, M. "The nature of laws". Canadian Journal of Philosophy, Vol. 7, Nr. 4, pp. 667-698, 1977.

. "Causation: A Realist Approach". Oxford: Oxford University Press, 1988. 